\title{
Enfermedad CLN8 congénita de lipofuscinosis neuronal ceroidea: un nuevo fenotipo
}

\author{
Favio Pesaola, Romina Kohan, Inés A. Cismondi, Norberto Guelbert, Patricia Pons, Ana M. Oller-Ramírez, \\ Inés Noher de Halac
}

Introducción. La enfermedad CLN8 es uno de los 13 tipos genéticos reconocidos de lipofuscinosis neuronal ceroidea, un grupo de trastornos neurodegenerativos de acumulación lisosómica, los más frecuentes en la infancia. La causan mutaciones en la proteína transmembrana CLN8 de 286 aminoácidos, cuya función se desconoce. Las variantes patológicas en el gen CLN8 se asociaron con dos fenotipos diferentes: la variante infantil tardía en individuos de diversos países alrededor del mundo, y la epilepsia progresiva con retraso mental, que aparece en pacientes finlandeses y turcos.

Caso clínico. Niña que mostró retraso psicomotor y demencia desde el nacimiento, convulsiones tonicoclónicas, mioclonía, ataxia con atrofia cerebelosa y muerte temprana a los 12 años. La microscopia electrónica de la piel mostró una mezcla de citosomas con patrones de depósitos osmiofílicos granulares, curvilíneos y de 'huella digital', y mitocondrias hipertrofiadas. Se encontraron dos variantes patológicas de ADN en el gen CLN8 (exón 2 c.1A>G; p.?/ exón 3 c.792C>G; p.Asn264Lys), lo que confirmó un genotipo heterocigoto compuesto.

Conclusión. Éste es el caso índice en América Latina para el nuevo fenotipo congénito de la enfermedad CLN8. La sospecha de esta patología debería sustentarse genéticamente en casos de síndrome neurodegenerativo con retraso psicomotor desde el nacimiento, dificultad del habla y convulsiones. El curso clínico incluye ataxia, atrofia cerebelosa y muerte temprana.

Palabras clave. América Latina. Caso índice. Enfermedad CLN8. Fenotipo congénito. Lipofuscinosis neuronal ceroidea. Mutación heterocigota compuesta.

\section{Introducción}

La enfermedad CLN8 es una de las 13 formas genéticas reconocidas de lipofuscinosis neuronal ceroidea, un grupo de trastornos neurodegenerativos hereditarios de acumulación lisosómica. Está causada por variantes en el gen CLN8 (MIM *607837) [1], el cual codifica para la proteína transmembrana CLN8 (CLN8p) de 286 aminoácidos, residente en el retículo endoplásmico [2]. El papel de CLN8p aún se desconoce; sin embargo, se ha asociado con lípidos [3]. Dos fenotipos están relacionados con mutaciones en el gen CLN8: epilepsia progresiva con retraso mental (EPRM), también conocida como síndrome de epilepsia del norte (OMIM 610003) [4], y una variante infantil tardía (vIT) (OMIM 600143) [5]. Las principales características de ambas variantes se resumen en la tabla. Ningún caso de CLN8 se ha descrito hasta la actualidad en América Latina, ni tampoco los fenotipos congénitos, infantil o adulto.

El objetivo de este trabajo es resignificar el valor de un enfoque clínico y genómico en el esclarecimiento de un caso de una enfermedad neurodegenerativa poco frecuente desde el nacimiento.

Los procedimientos desarrollados estuvieron de acuerdo con la Declaración Universal de Derechos
Humanos y Bioética de la Unesco de 2005, y la abuela (tutora) firmó un consentimiento informado aprobado por el Comité Interinstitucional de Ética de la Investigación en Salud (CIEIS-Córdoba, Argentina).

\section{Caso clínico}

Una de dos mellizas argentinas de padres no consanguíneos falleció a los 12 años. La madre murió debido a una cardiopatía y el padre abandonó la familia un tiempo antes de la consulta médica. La niña mostró retraso psicomotor y demencia desde el nacimiento, y convulsiones tonicoclónicas desde los 3 años. Pudo caminar sola a los 2 años y nunca desarrolló completamente el habla. Los exámenes de resonancia magnética y tomografía axial computarizada a los 6 años revelaron atrofia cerebelosa (Fig. 1). A los 7 años, la niña mostró ataxia grave, temblores y caídas constantes, y retraso neuromadurativo importante, aunque se mantenía conectada con sus tutores. Los estudios neurometabólicos realizados en CEMECO (ensayos cualitativos de oligosacáridos urinarios y aminoácidos plasmáticos, y mediciones cuantitativas de ácidos orgánicos urinarios) arrojaron resultados en el rango normal, y también los en-
Consejo Nacional de Investigaciones Científicas y Técnicas, CONICET (F. Pesaola, A.M. Oller-Ramírez, I. Noher de Halac). Programa de Investigación Traslacional de Lipofuscinosis Ceroidea Neuronal (F. Pesaola, A.M. Oller-Ramírez, I. Noher de Halac); Sección de Enfermedades Metabólicas Hereditarias (N. Guelbert); Hospital de Niños de Córdoba. Facultad de Odontología (R. Kohan, I.A. Cismondi); Centro de Miscroscopía Electrónica; Facultad de Ciencias Médicas (P. Pons); Universidad Nacional de Córdoba. Córdoba, Argentina.

Correspondencia:

Dr. Favio Pesaola. Programa de Investigación Traslacional de Lipofuscinosis Ceroidea Neuronal (NCLCEMECO). Hospital de Niños de Córdoba. Ferroviarios, 1250 X5014AKN Córdoba (Argentina).

E-mail:

favio.pesaola@gmail.com

Financiación:

I.N.H. es investigadora de CONICET y F.P. posee una beca de estudio de CONICET.

Agradecimientos:

K.B. Sims y W. Xin (Neurogenetics DNA Diagnostic Laboratory, Centre for Human Genetic Research, Massachusetts General Hospital, Boston, Estados Unidos), por colaborar en la secuenciación y búsqueda de mutaciones en el gen CLN8 de los pacientes.

Aceptado tras revisión externa: 20.07.18.

Cómo citar este artículo:

Pesaola F, Kohan R, Cismondi IA, Guelbert N, Pons P, Oller-Ramírez AM, et al. Enfermedad CLN8 congénita de lipofuscinosis neuronal ceroidea: un nuevo fenotipo. Rev Neurol 2019; 68: 155-9.

English version available at www.neurologia.com (c) 2019 Revista de Neurología 
Tabla. Comparación de las historias naturales de los fenotipos congénito, vIT y EPRM de CLN8.

\begin{tabular}{|c|c|c|c|c|}
\hline & & Congénito & vIT & EPRM \\
\hline \multicolumn{2}{|l|}{ Origen } & Argentina & $\begin{array}{l}\text { Varios países } \\
\text { alrededor del mundo }\end{array}$ & $\begin{array}{l}\text { Finlandia } \\
\text { y Turquía }\end{array}$ \\
\hline \multicolumn{2}{|c|}{ Edad de inicio } & Nacimiento & 2-7 años & 5-10 años \\
\hline \multicolumn{2}{|c|}{ Síntomas iniciales } & $\begin{array}{c}\text { Retraso psicomotor, } \\
\text { dificultades del } \\
\text { habla }\end{array}$ & $\begin{array}{l}\text { Convulsiones, mioclo- } \\
\text { nías o deterioro psico- } \\
\text { motor generalizado }\end{array}$ & $\begin{array}{l}\text { Convulsiones } \\
\text { tonicoclónicas }\end{array}$ \\
\hline \multirow{5}{*}{ Síntomas } & Convulsiones refractarias & Sí & Sí & Sí \\
\hline & Mioclonías & Sí & Sí & No \\
\hline & Deterioro motor & Sí & Sí & Sí \\
\hline & Discapacidad intelectual & Sí & Sí & Sí \\
\hline & Fallos en la visión & $?$ & Sí & No \\
\hline \multicolumn{2}{|c|}{ Resonancia magnética } & $\begin{array}{c}\text { Atrofia } \\
\text { cerebelosa }\end{array}$ & $\begin{array}{l}\text { Atrofia cortical } \\
\text { y cerebelosa }\end{array}$ & $\begin{array}{l}\text { Atrofia cortical } \\
\text { y cerebelosa }\end{array}$ \\
\hline \multicolumn{2}{|c|}{ Microscopía óptica } & $\begin{array}{l}\text { Sin linfocitos } \\
\text { vacuolados }\end{array}$ & - & $\begin{array}{c}\text { Neuronas globulares } \\
\text { sin vacuolas }\end{array}$ \\
\hline \multicolumn{2}{|c|}{$\begin{array}{l}\text { Microscopía electrónica } \\
\text { de transmisión }\end{array}$} & $\begin{array}{l}\text { Cuerpos en 'huella } \\
\text { digital', cuerpos } \\
\text { curvilíneos, GROD y } \\
\text { atrofia mitocondrial }\end{array}$ & $\begin{array}{c}\text { Cuerpos en 'huella } \\
\text { digital', cuerpos } \\
\text { curvilíneos y } \\
\text { raramente GROD }\end{array}$ & GROD \\
\hline \multicolumn{2}{|c|}{ Edad de fallecimiento } & 12 años & $\begin{array}{l}\text { Generalmente, an- } \\
\text { tes de los } 20 \text { años }\end{array}$ & $\begin{array}{l}\text { Generalmente, des- } \\
\text { pués de los } 50 \text { años }\end{array}$ \\
\hline \multicolumn{2}{|c|}{$\begin{array}{l}\text { Variantes genéticas } \\
\text { patológicas descritas }\end{array}$} & $\begin{array}{c}\text { c.1A }>\text { G, p.? } \\
.792 \text { C }>\text { G, p.Asn264lys }\end{array}$ & $\begin{array}{l}\text { Más de } 30 \text { mu- } \\
\text { taciones descritas }\end{array}$ & $\begin{array}{l}\text { c.70C }>\text { G, p.Arg24Cly } \\
\text { c.677T >C, p.Leu226Pro }\end{array}$ \\
\hline
\end{tabular}

EPRM: epilepsia progresiva con retraso mental; GROD: depósitos osmiofílicos granulares; vIT: variante infantil tardía. roidea en la población argentina. El estudio diagnóstico de todos los pacientes incorporados al Programa de Investigación Traslacional de Lipofuscinosis Ceroidea Neuronal en Argentina se realizó siguiendo el algoritmo previamente publicado [6]. Los métodos de microscopia electrónica de transmisión y ensayo enzimático se publicaron previamente [6-9].

\section{Análisis genómicos}

Se aisló ADN genómico de la sangre periférica usando Wizard DNA Purification Kit (Promega, Estados Unidos) siguiendo el protocolo del producto. La región codificante del gen CLN8 se amplificó por reacción en cadena de la polimerasa (oligos disponibles bajo pedido). Las secuenciaciones se realizaron en el University of Chicago Comprehensive Cancer Centre (Chicago, Estados Unidos) y en el Laboratorio de Diagnóstico Neurogenético del Centre for Human Genetic Research, ambos en un secuenciador Applied Biosystems 3730XL. Todas las secuencias controles del gen CLN8, tanto humano (RefSeq NM_018941, NP_061764) como de otras especies, fueron obtenidas de la base de datos Ensembl v. 90. Las variantes de ADN fueron excluidas de 100 individuos sanos de la población local (muestras provistas amablemente por el banco de sangre de la Universidad Nacional de Córdoba) usando el programa MEGA v. 5.05 [10] para los alineamientos. Las variantes proteínicas no sinónimas fueron validadas como de significación patológica por evaluación con los programas SIFT [11], PolyPhen [12] y Mutation Taster. Las variantes no codificantes fueron analizadas usando el programa RESCUE-ESE [13,14].

Las secuenciaciones mostraron los cambios c. $1 \mathrm{~A}>\mathrm{G}$ en el exón 2 y c.792C $>\mathrm{G}$ en el exón 3, ambos en estado heterocigoto. Además, se observaron otras dos variantes en el exón 1: c.-257G>C y c.-280 _-279insG. El cambio c.1A > G se encontró también en estado heterocigoto en la hermana melliza y la abuela materna, lo que estableció que este cambio proviene de la línea materna. El origen del cambio c.792C $>\mathrm{G}$ no pudo establecerse debido a la falta de muestras de la familia paterna.

Ninguna de las variantes c. $1 \mathrm{~A}>\mathrm{G}$ o c.792C $>\mathrm{G}$ se encontró en los 100 controles de la población local. La primera metionina de CLN8p está altamente conservada en todas las especies comparadas, a diferencia de la asparagina en la posición 264. El programa SIFT predijo ambas mutaciones como 'perjudiciales', PolyPhen las predijo como 'probablemente perjudiciales', y el programa Mutation Tester predijo ambas variantes como 'patológicas'. La va-

riante c.-280_-279insG en el exón 1 podría alterar el
Este trabajo es parte del estudio observacional de cohorte de la distribución de lipofuscinosis neuronal ce- 
Figura 1. Imágenes de resonancia magnética de la paciente a los 6 años. Se observa una formación quística en la fosa posterior que, según se revela en el plano sagital, parece conectarse con el cuarto ventrículo y se asocia con la dilatación del sistema ventricular supratentorial, que podría relacionarse con una variante del síndrome de Dandy-Walker.

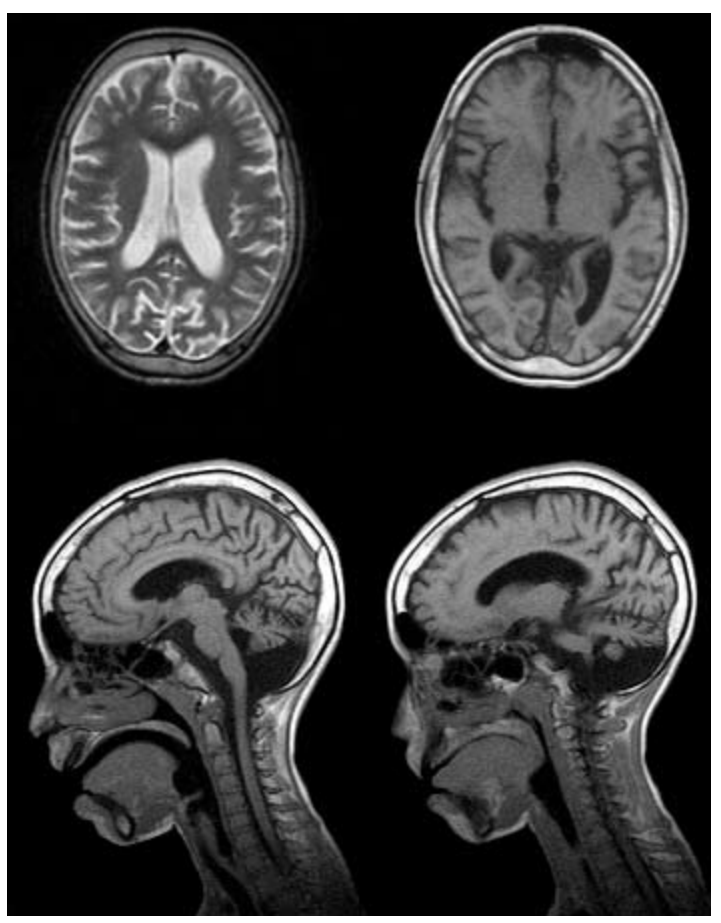

exonic splicing enhancer predicho 5'-GCAGAA-3' entre las bases - 280 y -275. De acuerdo con las recomendaciones de la Human Genome Variation Society, las mutaciones c.1A>G, p.? y c.792C>G, p.Asn264Lys deberían considerarse con significación patológica. Por otro lado, no fue posible confirmar la patogenicidad de las variantes c.-280_-279insG y c. $-257 \mathrm{G}>\mathrm{C}$.

La variante c. $1 \mathrm{~A}>\mathrm{G}$ elimina el codón de la primera metionina; por lo tanto, mueve el origen de traducción 'aguas abajo', causando un corrimiento del marco de lectura y un nuevo codón de parada, lo que genera un péptido de 22 aminoácidos. Un análisis de BLAST posterior no reveló una secuencia homóloga confiable para este polipéptido (datos no publicados). De acuerdo con la nomenclatura de mutaciones de la Human Genome Variation Society, esta mutación debería codificarse como c.1A>G, p.?. Son necesarios análisis posteriores para confirmar la expresión de este péptido aparentemente no funcional. Ambas variantes patógenas se incorpo-
Figura 2. Imágenes de microscopía electrónica de transmisión en una biopsia de piel. a, b) Diferentes perfiles de acumulación asociados con gránulos de lípidos; frecuentemente se observan mitocondrias hipertrofiadas en microscopía electrónica de transmisión de pacientes CLN8; c, d) Perfiles mixtos asociados con gránulos de lípidos; e, f) Perfiles mixtos asociados con mitocondrias vacuoladas hipertrofiadas; g) Gran número de mitocondrias hipertróficas; h) Citosoma con una acumulación compuesta de GROD. CB: cuerpos curvilíneos; FP: cuerpos en 'huella digital'; G: aparato de Golgi; GROD: depósitos osmiofílicos granulares; L: gránulo de lípidos; M: mitocondria; N: núcleo; PM: membrana plasmática.

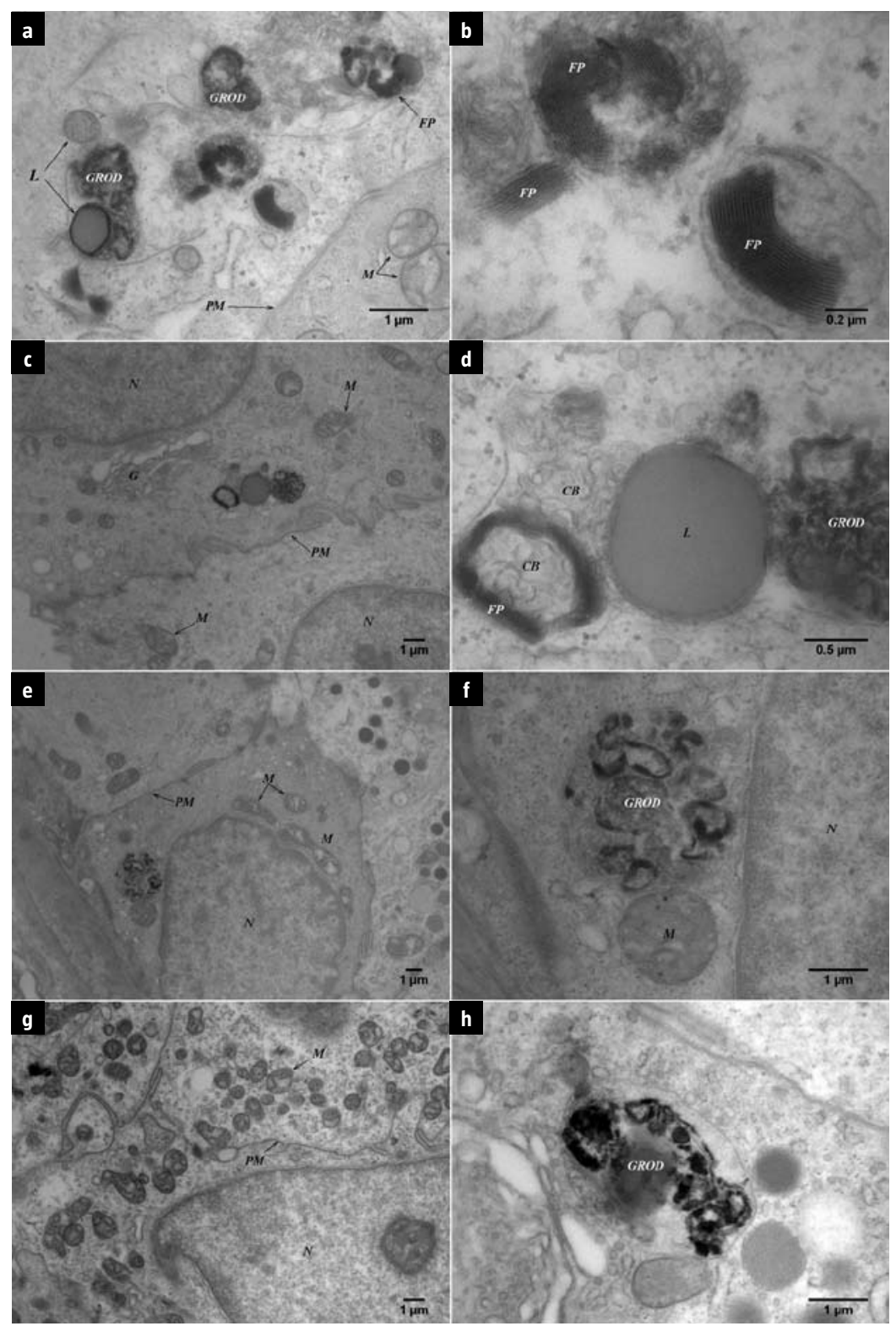

raron a NCL-Resource, la principal base de datos de mutaciones relacionadas con lipofuscinosis neuronal ceroidea, y a ClinVar (n. ${ }^{\circ}$ de acceso SCV000676930 y SCV000660961). 
Figura 3. Esquema del gen CLN8 y la proteína que indica la posición de ambas variantes patógenas encontradas en la paciente de este estudio.

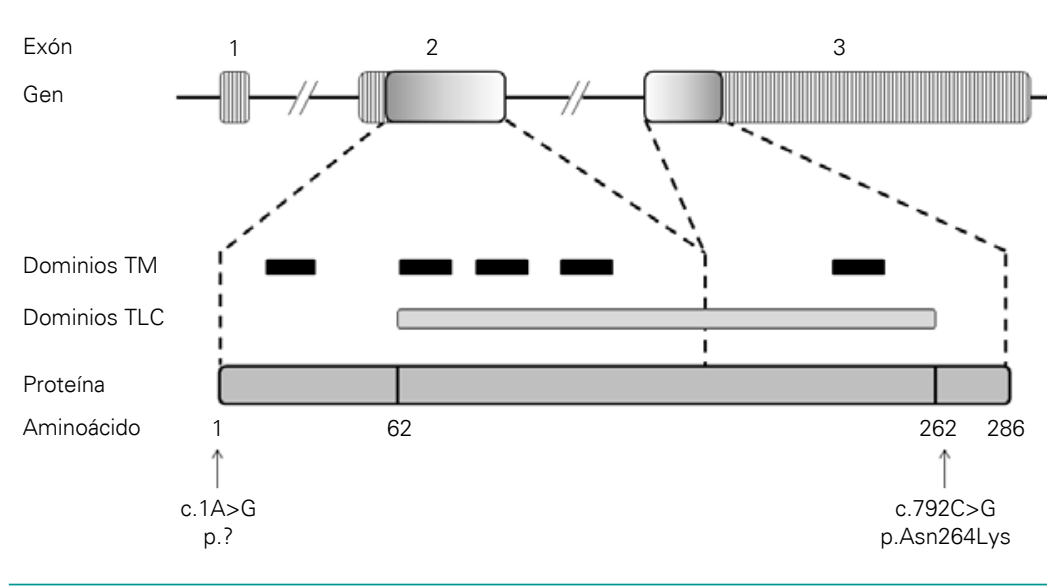

\section{Discusión}

La niña mostró un fenotipo congénito con dos variantes génicas en heterocigosis con significación patológica (exón 2 c.1A>G, p.? [rs143730802], y exón 3 c.792C>G, p.Asn264Lys [rs587779411]) (Fig. 3 ), y otras dos sin significación patológica (exón 1 c.-257G>C [rs71499040] y exón 1 c.-280_-279insG [rs71209699]).

La historia natural de la enfermedad se resume en la tabla en comparación con los fenotipos vIT y EPRM. El curso clínico se relaciona más con la forma vIT que con la EPRM, principalmente debido a su rápida evolución y a los movimientos mioclónicos. Los aspectos visuales no se examinaron. El inicio de los síntomas en el nacimiento es signo de un fenotipo congénito, el cual debe añadirse a los fenotipos aceptados para la enfermedad CLN8: vIT y EPRM [15]. Una relación genotipo/fenotipo congénito podría explicarse por la falta de expresión y disfunción de la proteína CLN8p. Hasta la actualidad, la enfermedad CLN10 (OMIM 610127) es la única forma de lipofuscinosis neuronal ceroidea congénita [16]. La variante c. $1 \mathrm{~A}>\mathrm{G}$ se citó previamente en un caso de CLN3 (enfermedad de Batten, OMIM 204200), sin una descripción del curso clínico del paciente [17].

Ambas variantes con significación patológica resultan nuevas para la enfermedad CLN8. La variante c.1A>G, p.? en el exón 2 se describió previamente en estado heterocigoto en el gen CLN8 de cuatro individuos como parte de una cohorte analizada en proyectos de secuenciación masiva: 1000 Genomes, UK 10K y NHLBI GO Exome Sequencing Project. Sin embargo, esta variante no se ha descrito como asociada a la enfermedad CLN8.

Según nuestro conocimiento, esta niña es el primer caso en América Latina de la enfermedad CLN8. Se informó de dos hermanos con el fenotipo CLN8 vIT de Canadá. Otro caso de heterocigosis compuesta en el gen $C L N 8$ (exon 2 c.1A>G, p.?/ exon 2 c.80T>C, p.Leu27Pro) se diagnosticó en Estados Unidos. Dos individuos sintomáticos de México y Argentina mostraron una variante heterocigota en CLN8, c.685C>G, p.Pro229Ala, y se les describió preliminarmente como sospechosos de la enfermedad CLN8 [18]. Posteriores análisis revelaron que esta variante de ADN es un polimorfismo local frecuente [19].

Individuos con el fenotipo CLN8 vIT [5,20,21] mostraron retraso en el desarrollo psicomotor a temprana edad (p. ej., comenzar a caminar después de los 15 meses o a hablar a los 3 años) y una progresiva pérdida de capacidades mentales y motoras más tarde, aproximadamente después de los 3,5 años. Todos fueron diagnosticados con el fenotipo CLN8 vIT, y tomaron como síntoma de inicio el que llevó a la consulta médica: marcha inestable, convulsiones, mioclonía o discapacidad intelectual. Previamente se discutió una correlación genotipo/fenotipo en CLN8 [5]. En concordancia con nuestros hallazgos, los pacientes que poseen síntomas neurológicos congénitos deberían ser agrupados en un fenotipo diferente: la forma congénita de la enfermedad CLN8.

En conclusión, hemos identificado el caso índice de CLN8 congénito en América Latina causado por variantes genéticas en heterocigosis: una variante genética patológica (exón $2 \mathrm{c} .1 \mathrm{~A}>\mathrm{G}, \mathrm{p}$.?) y una variante missense (exón 3 c.792C>G, p.Asn264Lys). Este caso expande la frontera de distribución de la enfermedad CLN8 a Argentina y amplía la información acerca de su espectro clínico, sumando un fenotipo congénito que debe sospecharse en recién nacidos con retraso psicomotor desde el nacimiento, dificultades del habla, síndrome convulsivo con posterior trastorno mioclónico del movimiento y ataxia.

La metodología de la reacción en cadena de la polimerasa y la secuenciación de Sanger 'de gen en gen' y 'de exón en exón' usada en este caso implicó mucho tiempo, con una deficiente relación costeefectividad. Este estudio sugiere que el uso sistemático de herramientas analíticas de la tecnología de secuenciación de nueva generación podría acelerar el reconocimiento de casos desafiantes de trastornos neurodegenerativos poco frecuentes en recién nacidos. El esclarecimiento de estos casos por análi- 
sis clinicogenómicos puede postularse como el enfoque más apropiado debido al incremento en la cobertura de lectura genética, reduciendo el tiempo y la odisea diagnóstica de las familias.

\section{Bibliografía}

1. Ranta S, Lehesjoki AE. Northern epilepsy, a new member of the NCL family. Neurol Sci 2000; 21 (Suppl 3): S43-7.

2 Lonka L, Kyttälä A, Ranta S, Jalanko A, Lehesjoki AE. The neuronal ceroid lipofuscinosis CLN8 membrane protein is a resident of the endoplasmic reticulum. Hum Mol Genet 2000; 9: 1691-7.

3 Winter E, Ponting CP. TRAM, LAG1 and CLN8: members of a novel family of lipid-sensing domains? Trends Biochem Sci 2002; 27: 381-3.

4 Herva R, Tyynelä J, Hirvasniemi A, Syrjäkallio-Ylitalo M, Haltia M. Northern epilepsy: a novel form of neuronal ceroidlipofuscinosis. Brain Pathol 2000; 10: 215-22.

5 Reinhardt K, Grapp M, Schlachter K, Brück W, Gärtner J, Steinfeld R. Novel CLN8 mutations confirm the clinical and ethnic diversity of late infantile neuronal ceroid lipofuscinosis. Clin Genet 2010; 77: 79-85.

6 Kohan R, Pesaola F, Guelbert N, Pons P, Oller-Ramírez AM, Rautenberg G, et al. The neuronal ceroid lipofuscinoses program: a translational research experience in Argentina. Biochim Biophys Acta 2015; 1852: 2300-11.

7 Kohan R, Noher de Halac I, Tapia-Anzolini V, Cismondi IA, Oller-Ramírez AM, Paschini-Capra A, et al. Palmitoyl protein thioesterase1 (PPT1) and tripeptidyl peptidase-I (TPP-I) are expressed in the human saliva. A reliable and non-invasive source for the diagnosis of infantile (CLN1) and late infantile (CLN2) neuronal ceroid lipofuscinoses. Clin Biochem 2005; 38: 492-4.

8 Kohan R, Cismondi IA, Dodelson de Kremer R, Muller VI, Guelbert N, Tapia Anzolini V, et al. An integrated strategy for the diagnosis of neuronal ceroid lipofuscinosis types 1 (CLN1) and 2 (CLN2) in eleven Latin American patients. Clin Genet 2009; 76: 372-82.

9 Kohan R, Carabelos MN, Xin W, Sims KB, Guelbert N, Cismondi IA, et al. Neuronal ceroid lipofuscinosis type CLN2: a new rationale for the construction of phenotypic subgroups based on a survey of 25 cases in South America. Gene 2013; 516: 114-21.

10 Tamura K, Peterson D, Peterson N, Stecher G, Nei M, Kumar S. MEGA5: molecular evolutionary genetics analysis using maximum likelihood, evolutionary distance, and maximum parsimony methods. Mol Biol Evol 2011; 28: 2731-9.

11 Kumar P, Henikoff S, Ng PC. Predicting the effects of coding non-synonymous variants on protein function using the SIFT algorithm. Nat Protoc 2009; 4: 1073-81.

12 Adzhubei IA, Schmidt S, Peshkin L, Ramensky VE, Gerasimova A, Bork P, et al. A method and server for predicting damaging missense mutations. Nat Methods 2010; 7: 248-9.

13 Fairbrother WG, Yeh RF, Sharp PA, Burge CB. Predictive identification of exonic splicing enhancers in human genes. Science 2002; 297: 1007-13.

14 Yeo G, Hoon S, Venkatesh B, Burge CB. Variation in sequence and organization of splicing regulatory elements in vertebrate genes. Proc Natl Acad Sci U S A 2004; 101: 15700-5.

15 Mole SE, Cotman SL. Genetics of the neuronal ceroid lipofuscinoses (Batten disease). Biochim Biophys Acta 2015; 1852: 2237-41.

16 Siintola E, Partanen S, Strömme P, Haapanen A, Haltia M, Maehlen J, et al. Cathepsin D deficiency underlies congenital human neuronal ceroid-lipofuscinosis. Brain 2006; 129: 1438-45.

17 Kousi M, Lehesjoki AE, Mole SE. Update of the mutation spectrum and clinical correlations of over 360 mutations in eight genes that underlie the neuronal ceroid lipofuscinoses. Hum Mutat 2012; 33: 42-63.

18 Cismondi IA. Estudio integral de las lipofuscinosis ceroideas neuronales genotipos CLN3, CLN5, CLN6, CLN7 y CLN8 en familias de Latinoamérica [tesis doctoral]. Córdoba, Argentina: Universidad de Córdoba; 2012.

19 Pesaola F. Lipofuscinosis ceroidea neuronal: caracterización molecular y bioinformática del gen CLN8 en pacientes de América Latina [tesis doctoral]. Córdoba, Argentina: Universidad de Córdoba; 2013.

20 Allen NM, O'hici B, Anderson GW, Nestor T, Lynch SA, King MD. Variant late-infantile neuronal ceroid lipofuscinosis due to a novel heterozygous CLN8 mutation and de novo 8p23.3 deletion. Clin Genet 2012; 81: 602-4.

21 Cannelli N, Cassandrini D, Bertini ES, Striano P, Fusco L, Gaggero R, et al. Novel mutations in CLN8 in Italian variant late infantile neuronal ceroid lipofuscinosis: another genetic hit in the Mediterranean. Neurogenetics 2006; 7: 111-7.

\section{Congenital CLN8 disease of neuronal ceroid lipofuscinosis: a novel phenotype}

Introduction. CLN8 disease is one of the thirteen recognized genetic types of neuronal ceroid lipofuscinosis, a group of neurodegenerative lysosomal storage disorders, most frequent in childhood. A putative 286 amino acids transmembrane CLN8 protein with unknown function is affected. Pathological variants in the CLN8 gene were associated with two different phenotypes: variant late-infantile in individuals from many countries worldwide, and epilepsy progressive with mental retardation, appearing in Finnish and Turkish subjects.

Case report. The girl showed psychomotor delay and dementia since birth, tonic-clonic seizures, myoclonus, ataxia with cerebellar atrophy, and early death at 12 years old. Electron microscopy of the skin showed mixed GROD, curvilinear, fingerprint cytosomes and mitochondrial hypertrophy. Two pathological DNA variants in the CLN8 gene (exon 2 c.1A>C; p.? / exon 3 c.792C>G; p.Asn264Lys) were found confirming a compound heterozygous genotype.

Conclusion. This case is the Latin American index for a new congenital phenotype of the CLN8 disease. The congenital phenotype has to be added to the clinical spectrum of the CLN8 disease. The suspicion of CLN8 disease should be genetically sustained in challenging cases of a neurodegenerative syndrome with psychomotor delay since birth, speech difficulty and seizures. The course includes ataxia, cerebellar atrophy, and early death.

Key words. CLN8 disease. Compound heterozygous mutation. Congenital phenotype. Index case. Latin America. Neuronal ceroid lipofuscinosis. 